\title{
On-chip OSNR Monitoring with Silicon Photonics Transparent Detector
}

\author{
Douglas Aguiar, Andrea Annoni, Emanuele Guglielmi, Francesco Zanetto, \\ Marco Sampietro, Andrea Melloni and Francesco Morichetti
}

\begin{abstract}
Non-invasive integrated detectors, named ContactLess Integrated Photonic Probe (CLIPP), are employed to demonstrate on-chip noise-independent power monitoring of optical channels and in-band Optical Signal to Noise Ratio (OSNR) measurement. The proposed technique is based on a two step lock-in demodulation of optical signals that are suitably labeled with low-modulation-index labels. We demonstrate OSNR measurement from 8 up to $27 \mathrm{~dB} / 0.1 \mathrm{~nm}$ on $10 \mathrm{Gbps}$ on-off keying (OOK) signals with a power level ranging from -25 up to $-15 \mathrm{dBm}$. This approach provides a promising tool for the monitoring of channels in reconfigurable optical networks with flexible channel allocation strategy, where the small channel separation makes the measurement of the in band OSNR challenging.
\end{abstract}

Index Terms-Silicon Photonics, Optical Signal to Noise Ratio, Optical Power Monitoring, Photonic Integrated Circuits.

\section{INTRODUCTION}

$\mathbf{O}$ $\mathrm{N}$-chip optical power monitors are essential components for the control and operation of complex photonic integrated circuits (PICs). Integrated detectors allow for diverse functions on a chip including, but not limited to, routing and switching of optical channels [1], [2], self configuring PICs [3], thermal tuning and wavelength locking of filters and modulators [4], [5]. In practical applications, most of these functions require the identification, monitoring and manipulation of optical signals regardless of the presence of other channels and/or optical noise. For applications where optical noise is propagating together with the signals, e.g. in systems with optical amplifiers such as Erbium Doped Fiber Amplifiers (EDFA) or Semiconductor Optical Amplifiers (SOA), the monitoring of the OSNR of the optical channel also becomes important. This measurement allows the identification of faults or defects on the system, permits the monitoring of the aging of the optical transmission channel and provides information on the Quality of Transmission (QoT) of the signal, which one can use to estimate the Bit Error Rate (BER) of the channel.

In this letter, we demonstrate that transparent integrated detectors, namely CLIPP [6], can measure the power of optical channels propagating in a silicon photonics $(\mathrm{SiP})$ waveguide regardless of the optical noise. To this aim, a 10 Gbps OOK optical channel is suitably labeled through a weak amplitude modulation that introduces negligible performance degradation. In addition, we show that the same technique can be used to measure on-chip the in-band OSNR of the optical channel.

Douglas Aguiar, Andrea Annoni, Emanuele Guglielmi, Francesco Zanetto, Marco Sampietro, Andrea Melloni and Francesco Morichetti are with the Dipartimento di Elettronica Informazione e Bioingegneria - Politecnico di Milano, Milan, 20133 Italy e-mail: (douglas.oliveiradeaguiar@polimi.it).

Manuscript received March xxx, 2017; revised April xxx, 2017.

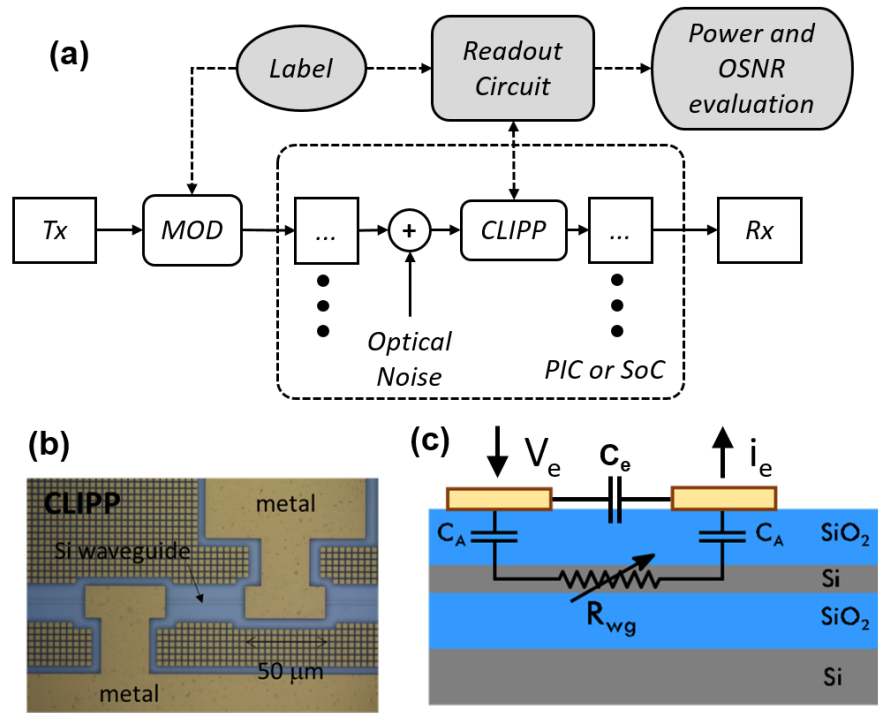

Fig. 1. a) Schematic of the proposed technique for the on-chip transparent measurement of optical channel power and the OSNR. b) Micrograph of the top view of the CLIPP device, and c) cross sectional scheme of the device showing its equivalent electrical circuit.

\section{Measurement technique}

The building blocks of the proposed measurement technique are shown in Fig. 1a). The PIC or System on Chip (SoC) inside the dashed lines can be as general as wished, possibly including one or more blocks before and/or after the CLIPP detector. These blocks can include both passive and active components that might add diverse kinds of optical noise such as Amplifier Spontaneous Emission (ASE), crosstalk due to backscatter and/or crosstalk from other propagating channels. As a basic concept of the proposed techniques, the signal power $P_{s}$ and the noise power $P_{n}$ can be discriminated from the total power $\left(P_{t}=P_{s}+P_{n}\right)$ because the signal is labeled with a weak modulation label, whereas the noise is not labeled. After recalling the main concepts of CLIPP detector in Sec. II-A, the signal labeling and detection techniques exploited in this work are described in Sec. II-B.

\section{A. Waveguide conductivity and optical power}

The CLIPP monitors the light intensity in a waveguide by measuring the change in the resistance $R_{w g}$ of the core segment between the two metal pads shown in Fig. 1b) [6]. This change is induced by free carrier generation, which in 
turn is due to photon absorption occurring in the semiconductor waveguide core. The change in the waveguide resistance versus the optical intensity exhibits different slopes at different power levels [7], depending on whether the dominant effect generating free carriers is either Surface State Absorption (SSA) or Two Photon Absorption (TPA) [8]. The general relation has a nonlinear dependence on the optical power and obtaining this relation is the first step to measure the optical power in the waveguide.

To measure the light dependent waveguide resistance, one needs an electrical path to access the waveguide resistance $R_{w g}$. As shown in Fig. 1c), the CLIPP device provides a non-invasive (contactless) access through a capacitive coupling $\left(C_{A}\right)$. The measurement of the device admittance is achieved via the two electrical signals $V_{e}$ and $i_{e}$ [6]. The relation between the CLIPP admittance and the waveguide resistance is given by

$$
Y_{\text {clipp }}(s)=s C_{e}+\frac{1}{R_{w g}} \frac{s}{s+2 / R_{w g} C_{A}}
$$

where $C_{e}$ is the parasitic capacitance between the two CLIPP terminals. To eliminate the dependence on $C_{e}$, which can be considered light independent, a dark-state reference measurement of $Y_{\text {clipp }}$ is performed without light propagating in the waveguide. By working with a reading frequency higher than the natural frequency of the CLIPP, $f_{e}=1 / \pi R_{w g} C_{A}$, the magnitude of the variation on the complex admittance, $\Delta Y_{\text {clipp }}$, provides directly the variation of the waveguide conductance $\Delta G_{w g}$. For $\mathrm{mW}$ range optical power in the waveguide, the conductance change $\Delta G_{w g}$ is tipically in the nano Siemens scale [6]. To measure such small values, a standard low noise trans-impedance amplifier (TIA) can be used and its output is applied to a lock-in demodulator. The output voltage of this lock-in demodulator, hereforth called the CLIPP demodulator, when subtracted from the reference darkstate voltage provides the absolute variation on the waveguide conductance $\Delta G_{w g}$.

\section{B. Channel labeling}

To measure channel powers independently of either multiple optical signals or optical noise propagating simultaneously in a waveguide, one can combine the use of channel labeling and CLIPP detectors. To this aim, on the transmitter side, the channel is intensity modulated with a frequency $f_{q}$ below the reading frequency of the CLIPP [2]. To not degrade the performance of the transmitted channel, a low modulation index $\mu(<8 \%)$ shall be used [8]. This can be achieved with a Mach-Zehnder (MZ) modulator driven with an amplitude much smaller than its $V_{\pi}$, as shown in Fig. 1a). The signal labeling can be performed either directly on-chip, by using thermally tuned MZ modulators [2] or with external modulators close to the transmitter.

To obtain the label power measurement one needs to add a label demodulation stage after the output of the CLIPP demodulator. The output voltage $V_{\text {out }, 2}$ of this second lockin demodulator is proportional to the change in the waveguide conductance $\Delta G_{\text {label }}$ which is due to the labeled signal. Since the label is simply an amplitude modulation of the signal, the

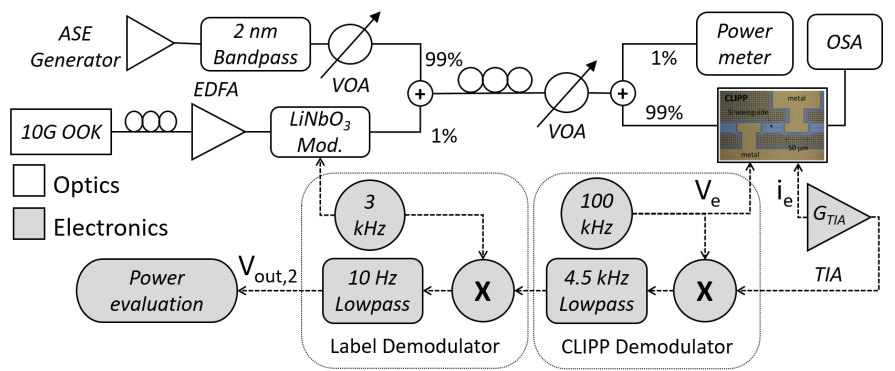

Fig. 2. Block diagram of the optical (white) and electrical (grey) setup used in the experiments.

relation between the signal power, $P_{s}$, and the label amplitude is given by the modulation index $\mu$. As a result, the relation between $\Delta G_{\text {label }}$, i.e. the optical power of the signal that was labeled $\left(P_{s}\right)$, and $V_{\text {out }, 2}$ is given by

$$
\Delta G_{\text {label }}=\frac{V_{\text {out }, 2}}{G_{T I A} V_{e} \mu \gamma}
$$

where $G_{T I A}$ the gain of the TIA and $\gamma$ is the slope of the Optical Power vs. $\Delta G$ relation (Eq. (3)), to be discussed in Section IV. The slope term appears in this relation since the label amplitude is proportional to the derivative of Eq. (3). The bandwidth of the label demodulation stage ultimately determines the tradeoff between the response time and the noise level of the signal power detection.

\section{EXPERIMENTAL SETUP}

In the experiments, each CLIPP pad is made of a $20 \times 50 \mu \mathrm{m}^{2}$ metal electrode deposited on top of a straight SiP channel waveguide. The two pads are spaced by $50 \mu \mathrm{m}$. The core has a rectangular cross section of $480 \times 220 \mathrm{~nm}^{2}$ and is buried in $600 \mathrm{~nm}$ thick $\mathrm{SiO}_{2}$ upper cladding.

The experimental setup is shown in Fig. 2. Since the addition of the label must not impair the transmitted signals, a label with a $7 \%$ modulation index at $f_{q}=3 \mathrm{kHz}$ is applied to a $10 \mathrm{Gbps}$ OOK signal, with carrier wavelength 1558.3 $\mathrm{nm}$, by a commercial $\mathrm{LiNbO}_{3} \mathrm{MZ}$ modulator biased at $3 \mathrm{~dB}$ transmission. An ASE noise source is coupled to this signal via a 90/10 fiber coupler, after being filtered by a $2 \mathrm{~nm}$ tunable filter. Two variable optical attenuators (VOAs) are used to control respectively the off-chip OSNR level between $5 \mathrm{~dB} / 0.1 \mathrm{~nm}$ and $24 \mathrm{~dB} / 0.1 \mathrm{~nm}$, and the signal power $P_{\text {signal }}$ from $-25 \mathrm{dBm}$ up to $-15 \mathrm{dBm}$. Since the noisy signal is coupled to the $\mathrm{SiP}$ chip through polarization selective grating couplers, the on-chip OSNR level is $3 \mathrm{~dB}$ higher than the offchip OSNR. As the ASE signal is not labeled, the second demodulation of the CLIPP at the label frequency $f_{q}$ can provide direct information on the signal power. It should also be noted that to compute the OSNR in $\mathrm{dB} / 0.1 \mathrm{~nm}$ the ratio between total noise power and the noise at $0.1 \mathrm{~nm}$ resolution bandwidth (RBW) should be known. In the experiments the ratio is $15.4 \mathrm{~dB}$. For systems with several noise sources, channels and filtering elements, an optical demultiplexer can be added to extract the channel to be monitored and a flat spectral portion of the noise. 


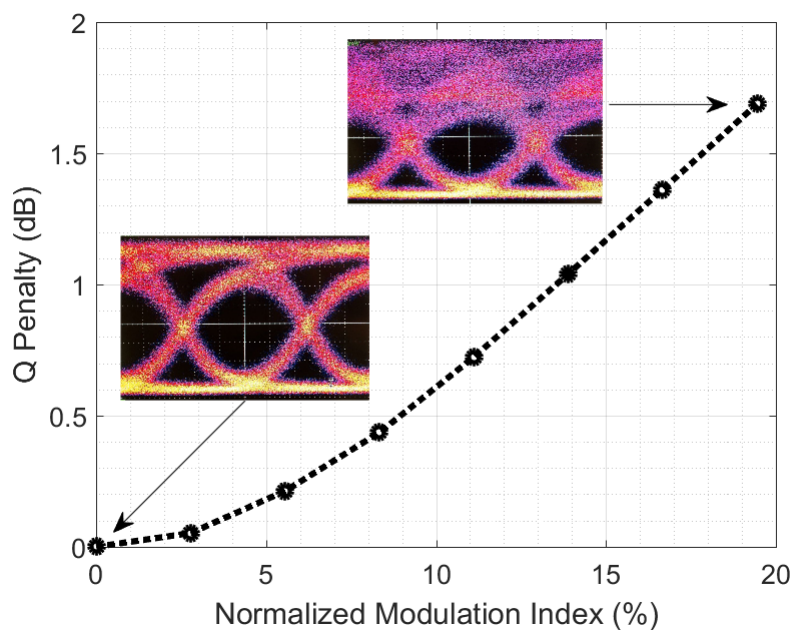

Fig. 3. Measurement of the $\mathrm{Q}$ factor penalty due to the insertion of a label on a $10 \mathrm{Gbps}$ OOK signal.

For the CLIPP readout circuit, one electrode of the CLIPP is driven with a sinusoidal voltage $\mathrm{V}_{e}=6 \mathrm{~V}_{\text {peak }}$ at $f_{e}=$ $100 \mathrm{kHz}$, while the second electrode reads the current signal $i_{e}$ that contains information on the light intensity inside the waveguide. The current is then converted to voltage via a low noise TIA and detected with a lock-in amplifier whose low pass filter has a $3 \mathrm{~dB}$ bandwidth of $4.5 \mathrm{kHz}$. The label demodulation is then applied to the output of the CLIPP demodulator, at the label frequency of $3 \mathrm{kHz}$ and filtered with a lowpass narrow bandwidth filter with a $3 \mathrm{~dB}$ bandwidth of $10 \mathrm{~Hz}$. This narrow bandwidth allows a smaller standard deviation in the OSNR measurement.

\section{RESUlts}

To quantify the penalty induced by the label, the $\mathrm{Q}$ factor of the $10 \mathrm{Gbps}$ OOK signal was measured as a function of the modulation index $\mu$. Figure 3 shows that when the intensity of the label increases, the penalty in the $\mathrm{Q}$ factor also increases. The two eye diagrams shown in the insets correspond to the label-free signal $(\mathrm{Q}=7.7 \mathrm{dBQ})$ and to a label amplitude $\mu 19 \%(\mathrm{Q}=6.0 \mathrm{dBQ})$. To keep the $\mathrm{Q}$ penalty below $0.5 \mathrm{dBQ}$, a modulation index $\mu=7 \%$ is used for the reported measurements.

The measurements of the total power $P_{t}$ propagating in the waveguide was carried out by using either a $\mathrm{CW}$ source at a wavelength of $1558.35 \mathrm{~nm}$ or ASE noise (1530 nm to $1560 \mathrm{~nm}$ ). Results in Fig. 4 show that both sources generate the same conductance variation because of the broadband response of the CLIPP detector [9]. The accuracy of the measurement is related to the electrical bandwidth of the lock-in amplifier, i.e. its low pass filter. As shown in the inset of the figure, the standard deviation of the measured $\Delta G$ decreases from $1 \mathrm{nS}$ to $0.01 \mathrm{nS}$ when the bandwidth is narrowed from $100 \mathrm{~Hz}$ to $1 \mathrm{~Hz}$.

The measured points were fitted with a power law relation in the form of

$$
\Delta G=\alpha+\beta P_{t}^{\gamma}
$$

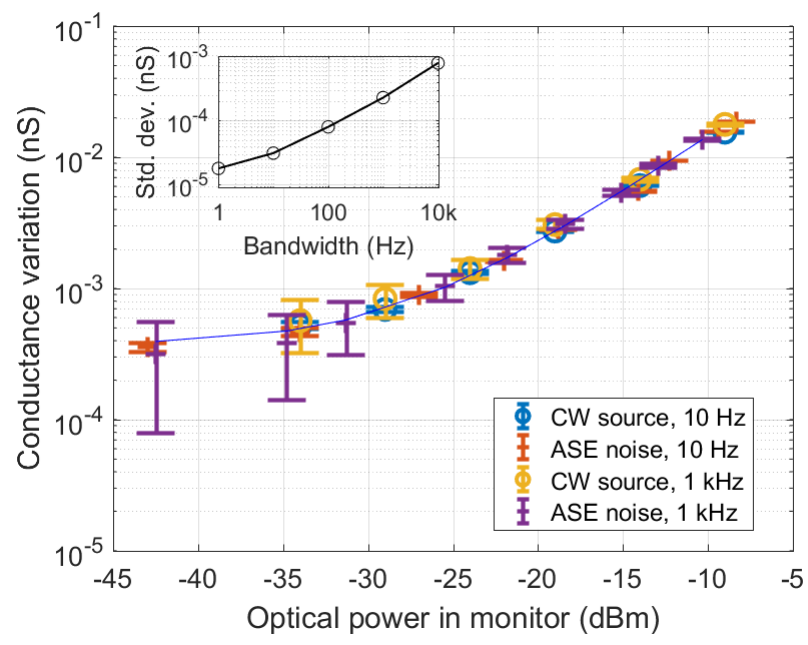

Fig. 4. Measured conductance variation $\Delta G$ as a function of total power $P_{t}$ in the silicon waveguide for CW and ASE signals at the CLIPP demodulator. The CLIPP is demodulated using two filter bandwidths $(10 \mathrm{~Hz}$ and $1 \mathrm{kHz})$ of the lock-in amplifier. The standard deviation of the measured $\Delta G$ versus the lock-in bandwidth is shown in the inset.

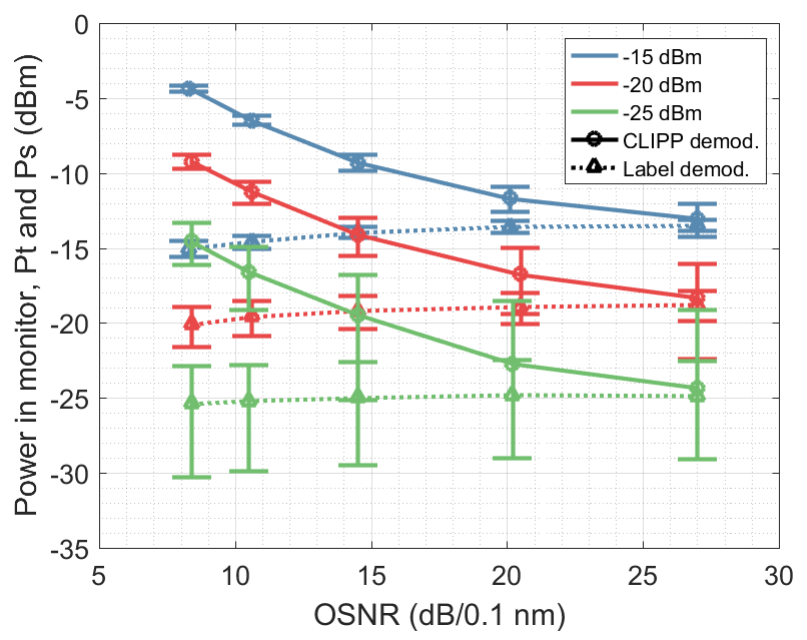

Fig. 5. Measured total power $P_{t}$ (solid lines, after CLIPP demodulation) and signal power $P_{s}$ (dashed lines, after label demodulation) of a $10 \mathrm{Gbps}$ OOK signal, for three different optical power levels $(-15 \mathrm{dBm}$ green, $-20 \mathrm{dBm}$ red, $-25 \mathrm{dBm}$ blue) in the silicon waveguide.

where $P_{t}$ is the power in the monitor in $\mathrm{dBm}$, and $\Delta G$ the waveguide conductivity variation in $\mathrm{nS}$, with $\alpha=3.7 \times 10^{-4}$, $\beta=0.073$ and $\gamma=0.87$. The blue line in Fig. 4 shows the fitted curve according to Eq. (3). The sublinear dependence of the conductance variation versus the optical power $(\gamma<1)$ results from a power dependent recombination time of the photogenerated carriers [6], [8]. At low power, the slope changes in agreement with other observations on photoconductiity [7]. This change in the $\Delta G$ vs $P_{t}$ slope shall be considered when computing the ratio between the label and total powers.

Figure 5 shows that the dual demodulation technique can be used to identify the channel power $P_{s}$ amid the total power $P_{t}$ of a noisy signal. Three signal power levels are considered, namely $-15,-20 \mathrm{dBm}$ and $-25 \mathrm{dBm}$ and the OSNR is varied by changing the ASE level. The solid lines $\left(P_{t}\right)$ are the output of 


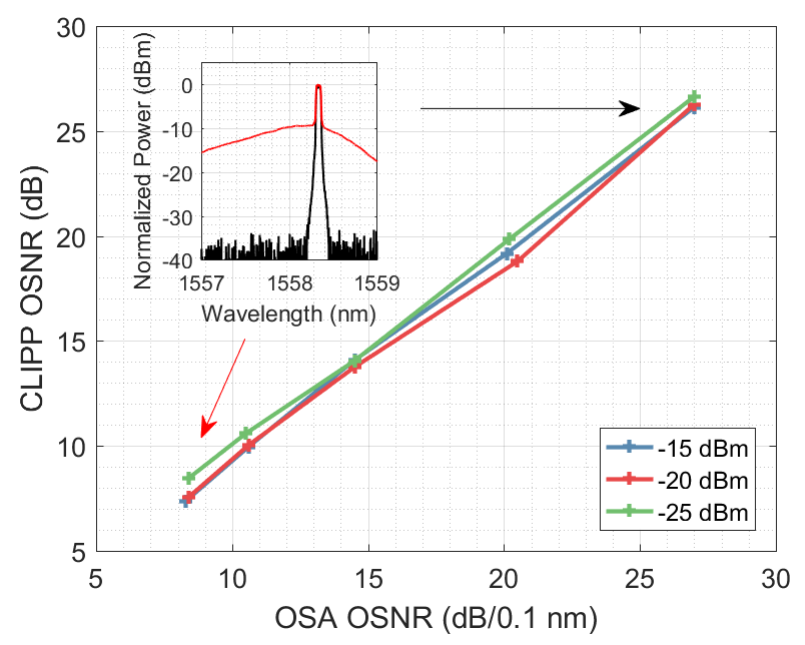

Fig. 6. Comparison between the OSNR measured on a $10 \mathrm{Gbps}$ OOK channel with an external OSA and the OSNR measured on-chip with the proposed technique. The three curves correspond to different values of the signal power $P_{s}=-15 \mathrm{dBm}$ (blue), $-20 \mathrm{dBm}$ (red), and $-25 \mathrm{dBm}$ (green).

the CLIPP demodulation at $f_{e}=100 \mathrm{kHz}$ filtered by the low pass filter with $4.5 \mathrm{kHz}$ bandwidth. The dashed lines $\left(P_{s}\right)$ are the output after the label demodulation at $f_{e}=3 \mathrm{kHz}$ filtered by the low pass filter with $10 \mathrm{~Hz}$ bandwidth, which does not include the in-band noise component of the signal. The error bars show the standard deviation of the measurements and the markers indicate the average values. It should be noted that the measurement of $P_{s}$ is independent of OSNR, thus allowing for an accurate measurement of the signal power regardless of the noise level. Since the power of the label $(\mu=$ $7 \%$ ) is more than $10 \mathrm{~dB}$ lower than the average power of the signal, the error bars in the measurement of $P_{s}$ after the label demodulation are larger than those of Fig. 4 (measured after CLIPP demodulation only), specially at the lowest power level of $-25 \mathrm{dBm}$. As expected, at high OSNR values the measured $P_{t}$ converge to the noise-free value given by $P_{s}$.

From the measurement of $P_{t}$ and $P_{s}$, the OSNR of the 10 Gbps channel can be directly derived. The curves of Fig. 6, obtained from the data of Fig. 5, show a good agreement between the OSNR measured with an external optical spectrum analyzer (OSA) and the signals provided by the CLIPP, in the measured OSNR range. A small deviation in the measurement of the OSNR $(< \pm 1 \mathrm{~dB})$ is observed with respect to the signal power $P_{s}$. The inset shows the optical spectrum at $0.1 \mathrm{~nm}$ of RBW of the measured signal in the low and high OSNR regions. These results show that the dual demodulation technique is robust and enables accurate on-chip measurement of in-band OSNR.

\section{CONCLUSiOnS}

We have proposed a technique to measure the in-band OSNR of a channel on a SiP chip by using transparent integrated monitor. OSNR values from 8 up to $27 \mathrm{~dB} / 0.1 \mathrm{~nm}$ were measured on a $10 \mathrm{Gbps}$ channel, with less than $1 \mathrm{~dB}$ deviation with respect to the measurement provided by an external OSA. Low dependence on the signal power, in the range between
-25 and $-15 \mathrm{dBm}$, was observed. The technique exploits the labeling of the optical signal with a weak modulation label, which adds a very small system penalty in the quality of the channel $(<0.4 \mathrm{dBQ}$ for $10 \mathrm{Gbps}$ OOK). In addition to the OSNR, the technique is able to provide a noise insensitive measurement of the optical power of noisy signals.

The presented approach, here employed for monitoring a 10 Gbps OOK channel, can be applied to optical signals with arbitrary bandwidths and amplitude/phase modulation formats, which can be also multiplexed in space, wavelength, polarization and/or angular momentum. In addition to the SiP platform employed in this work, this technique can also be implemented in III-V platforms, where the CLIPP functionality has been demonstrated [10], where it could be effectively used for a local noise level monitoring of integrated optical amplifiers. The labeling and detection method presented here does not strictly require the CLIPP detectors and could be implemented with standard photodiodes, yet requiring a portion of the optical power to be tapped out of the waveguide, as well as other non-invasive monitoring devices [11]. Finally, promising applications are expected for the monitoring of channels in reconfigurable optical networks with flexible channel allocation, where the small channel separation makes the measurement of the in-band OSNR challenging.

\section{ACKNOWLEDGMENT}

This work has been supported in part by the European Commission through the H2020 project ICT-STREAMS (Contract No. 688172), by the Erasmus Mundus Action 2 programme through the Sustain-T project and by Fondazione Cariplo under Project ACTIO (Rif. 2016-0881).

\section{REFERENCES}

[1] L. Qiao, W. Tang, and T. Chu, " $32 \times 32$ silicon electro-optic switch with built-in monitors and balanced-status units," Scientific Reports, vol. 7, 2017.

[2] A. Annoni et al., "Automated routing and control of silicon photonic switch fabrics," IEEE Journal of Selected Topics in Quantum Electronics, vol. 22, no. 6, pp. 169-176, Nov 2016.

[3] D. A. Miller, "Self-configuring universal linear optical component [invited]," Photonics Research, vol. 1, no. 1, pp. 1-15, 2013.

[4] K. Padmaraju et al., "Wavelength locking and thermally stabilizing microring resonators using dithering signals," J. Lightwave Technol., vol. 32, no. 3, pp. 505-512, Feb 2014.

[5] C. Sun et al., "A $45 \mathrm{~nm}$ cmos-soi monolithic photonics platform with bit-statistics-based resonant microring thermal tuning," IEEE Journal of Solid-State Circuits, vol. 51, no. 4, pp. 893-907, April 2016.

[6] F. Morichetti et al., "Non-invasive on-chip light observation by contactless waveguide conductivity monitoring," IEEE Journal of Selected Topics in Quantum Electronics, vol. 20, no. 4, pp. 292-301, 2014.

[7] S. S. Li, Semiconductor Physical Electronics. Springer, 2006.

[8] S. Grillanda and F. Morichetti, "Light-induced metal-like surface of silicon photonic waveguides," Nature communications, vol. 6, 2015.

[9] M. Carminati et al., "Design guidelines for contactless integrated photonic probes in dense photonic circuits," Journal of Lightwave Technology, vol. PP, no. 99, pp. 1-1, 2017.

[10] D. Melati et al., "Contactless integrated photonic probe for light monitoring in indium phosphide-based devices," IET Optoelectronics, vol. 9, pp. 146-150(4), August 2015. [Online]. Available: http: //digital-library.theiet.org/content/journals/10.1049/iet-opt.2014.0159

[11] D. Li, L. Zhou, L. Lu, and J. Chen, "Optical power monitoring with ultrahigh sensitivity in silicon waveguides and ring resonators," IEEE Photonics Journal, vol. 9, no. 5, pp. 1-10, Oct 2017. 